\title{
Quantum disorder and Griffiths singularities in bond-diluted two-dimensional Heisenberg antiferromagnets
}

\author{
Rong Yu, ${ }^{1}$ Tommaso Roscilde ${ }^{*},{ }^{1}$ and Stephan Haas ${ }^{1}$ \\ ${ }^{1}$ Department of Physics and Astronomy, University of Southern California, Los Angeles, CA 90089-0484
}

\begin{abstract}
We investigate quantum phase transitions in the spin-1/2 Heisenberg antiferromagnet on square lattices with inhomogeneous bond dilution. It is shown that quantum fluctuations can be continuously tuned by inhomogeneous bond dilution, eventually leading to the destruction of long-range magnetic order on the percolating cluster. Two multicritical points are identified at which the magnetic transition separates from the percolation transition, introducing a novel quantum phase transition. Beyond these multicritical points a quantum-disordered phase appears, characterized by an infinite percolating cluster with short ranged antiferromagnetic order. In this phase, the low-temperature uniform susceptibility diverges algebraically with non-universal exponents. This is a signature that the novel quantum-disordered phase is a quantum Griffiths phase, as also directly confirmed by the statistical distribution of local gaps. This study thus presents evidence of a genuine quantum Griffiths phenomenon in a two-dimensional Heisenberg antiferromagnet.
\end{abstract}

PACS numbers: 75.10.Jm, 75.10.Nr, 75.40.Mg, 64.60.Ak

\section{INTRODUCTION}

Quantum phase transitions in low-dimensional quantum Heisenberg antiferromagnets (QHAFs) have been the subject of extensive investigations during the last two decades 1.2 . The transition from a renormalized classical to a quantum-disordered state can be triggered by various parameters, including lattice dimerization, frustration and applied field. More recently, special interest has focused on phase transitions driven by geometric randomness of the lattice $e^{3.4}$, including site and bond disorder. Strong geometric disorder not only breaks translational invariance and perturbs the ground state of the pure system, but it can also destabilize renormalized classical phases with long-range order (LRO) and drive the system to novel disordered phases.

Various one-dimensional QHAFs with bond disorder have been found to display unconventional quantum phases.6. For example, the undimerized QHAF chain is driven into a random-singlet phase $\underline{\underline{6}}^{\underline{6}}$ with algebraically decaying spin-spin correlations. The low temperature susceptibility diverges as $1 /\left[\operatorname{Tlog}^{2} T\right]$ in this phase, independent of the details of the bond disorder. In contrast, a dimerized chain shows a quantum Griffiths phase ${ }^{\underline{\underline{T}}}$ beyond a critical disorder strength, with exponentially decaying spin-spin correlations and a non-universal powerlaw divergent susceptibility. In two dimensions, the clean QHAF develops antiferromagnetic (Néel) LRO at zero temperature ${ }^{8}$. This introduces the intriguing possibility of a genuine order-disorder transition, driven by lattice randomness, i.e. from the Néel phase into one of the above unconventional disordered phases ${ }^{9}$.

Site and bond dilution of the square lattice QHAF have been the focus of several recent studies,3.13,14.15,

*Current address: Max-Planck-Institut für Quantenoptik, HansKopfermann-str. 1, D-85748 Germany. motivated by experiments on antiferromagnetic cuprates doped with nonmagnetic impurities 3 3.16,17. From a geometric point of view, bond and site dilution reduce the connectivity of the lattice, ultimately leading to a percolative phase transition ${ }^{18}$ beyond which the system is broken up into finite clusters. In a classical spin system, this percolation transition is coupled to a magnetic transition with the same critical exponents since spontaneous magnetic order cannot survive beyond the percolation threshold. In a quantum spin system, on the other hand, a progressive reduction of the lattice connectivity enhances quantum fluctuations in a continuous fashion, raising the possibility of quantum destruction of magnetic order before the percolation threshold is reached. However, recent studies of homogeneously site- and bonddiluted QHAFs on the square lattice $\frac{3.4 .19 .20}{}$ found that the magnetic transition takes place exactly at the percolation threshold, due to the fact that the percolating cluster at threshold shows LRO ${ }^{4}$. The critical exponents of the correlation length and of the order parameter, $\nu$ and $\beta$, are found to take their classical percolation values ${ }^{4.21}$. Therefore, in this case the magnetic transition is completely dominated by classical percolation ${ }^{22}$.

Alternative to the above classical percolation picture is the quantum percolation mechanism, recently demonstrated in a number of model systems $23,24,25,26$. This scenario is based on the fact that spins involved in locally strongly fluctuating quantum states, such as dimer singlets and resonating valence bonds (RVBs), are weakly correlated with the remainder of the system. In a random network of spins, the local strongly fluctuating states create weak links with small spin-spin correlations. If these weak links are part of the backbone of the percolating cluster, they can prevent the percolating cluster from developing long-range order. Therefore, if lattice dilution favors the local formation of such states, it is possible to drive the system towards a quantum disordered state before the percolation threshold is reached, thus decoupling percolation from magnetic ordering. 
In this work we numerically scrutinize this quantum percolation scenario in the $S=1 / 2 \mathrm{QHAF}$ on a square lattice with inhomogeneous bond dilution 27 . The inhomogeneous character of lattice randomness is the key ingredient to enhance quantum fluctuations, favoring the local formation of RVB/dimer-singlet states. Based on large-scale Quantum Monte Carlo (QMC) simulations, we observe that the classical percolation scenario is preserved for moderate inhomogeneity, up to a multicritical point beyond which the magnetic transition deviates from the percolation threshold. At this point, the quantum percolation scenario sets in, leading to a novel quantum-disordered phase. Such a phase has very unconventional features, i.e. a finite correlation length but a gapless excitation spectrum, and a diverging uniform susceptibility at zero temperature with a non-universal exponent. These signatures allow us to identify this phase with a genuine two-dimensional quantum Griffiths phase ${ }^{7,28,29}$.

This paper is organized as follows. In Sec. Ithe model is described, and in Sec. III some technical aspects of the simulations are reviewed. In Sec. IV we discuss in detail the fate of antiferromagnetic order on the percolating cluster upon tuning the inhomogeneity of bond disorder. The complete phase diagram of the model is discussed in Sec. V] Sec. VI deals with the emergence of the quantum-disordered phase in the limit of randomly coupled ladders, whereas Sec. VII is dedicated to the novel ground state properties of the quantum-disordered regime. Conclusions are drawn in Sec. VIII

\section{INHOMOGENEOUS BOND DILUTION OF THE QHAF ON THE SQUARE LATTICE}

We investigate the ground state and thermodynamics of the $S=1 / 2 \mathrm{QHAF}$ on a two-dimensional square lattice with inhomogeneous bond dilution. The Hamiltonian of this system is given by

$$
\mathcal{H}=J \sum_{\langle i j\rangle \in D} \epsilon_{D}^{(i j)} \boldsymbol{S}_{i} \cdot \boldsymbol{S}_{j}+J \sum_{\langle l m\rangle \in L} \epsilon_{L}^{(l m)} \boldsymbol{S}_{l} \cdot \boldsymbol{S}_{m}
$$

The sums run over the dimer $(D)$ and ladder (interdimer) $(L)$ bonds, as indicated in Fig. 11(a). The exchange couplings are taken to be equal and antiferromagnetic $(J>0) . \epsilon_{\alpha}(\alpha=D, L)$ is a random variable drawn from a bimodal distribution taking values 1 ('on') or 0 ('off'). The inhomogeneity of this model is of purely statistical nature, stemming from the different probabilities of assigning the 'on'-state $\left(\epsilon_{i}=1\right)$ to the dimer bonds versus the ladder bonds. For $D$ bonds this probability is denoted by $p\left(\epsilon_{D}=1\right)=P_{D}$, whereas for $L$ bonds it is denoted by $p\left(\epsilon_{L}=1\right)=P_{L}$. The special case $P_{D}=P_{L}$ represents the previously studied homogeneous bond-dilution problem ${ }^{4.18}$. In the general inhomogeneous case $P_{D} \neq P_{L}$, two limits are noteworthy (see Fig. 1(b)-(c)): the case $P_{D}=1, P_{L} \in[0,1]$ corresponds
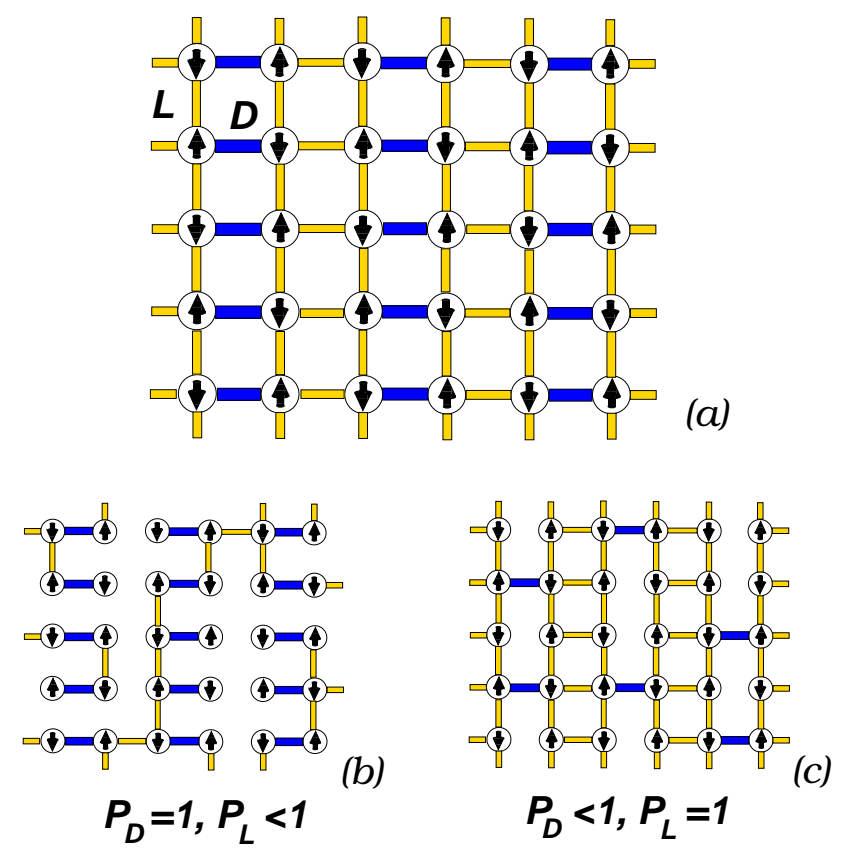

FIG. 1: (color online) (a) Decomposition of the square lattice into dimer $(D)$ and ladder $(L)$ bonds; (b) randomly coupled dimer limit; (c) randomly coupled ladder limit.
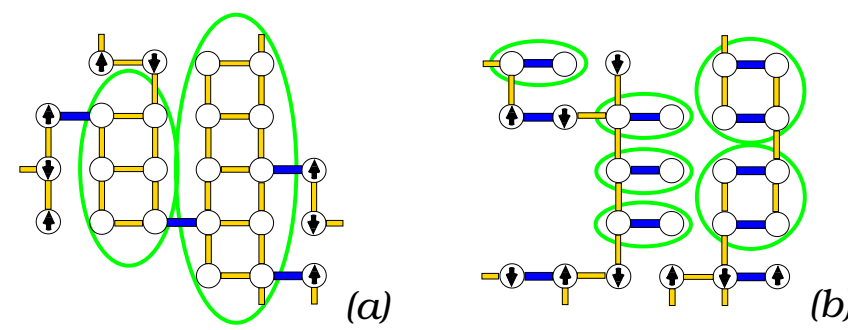

(b)

FIG. 2: (color online) Inhomogeneous bond dilution favors the formation of local strongly fluctuating quantum states. The green ellipses indicate such local dimer-singlet, plaquettesinglet and ladder-like RVB states: (a) $P_{L}>P_{D}$; (b) $P_{D}>$ $P_{L}$.

to a system of randomly coupled dimers, and the opposite case $P_{L}=1, P_{D} \in[0,1]$ corresponds to randomly coupled ladders. The degree of inhomogeneity can be parametrized by introducing a variable

$$
\theta=\arctan \left(\frac{1-P_{L}}{1-P_{D}}\right)
$$

such that the limiting cases of randomly coupled ladders, homogeneous bond dilution and randomly coupled dimers correspond to $\theta=0, \pi / 4$, and $\pi / 2$ respectively.

It is evident that the inhomogeneous nature of bond dilution in the lattice enhances quantum fluctuations in the magnetic Hamiltonian. In the strongly inhomogeneous limits $\theta \rightarrow 0, \pi / 2$ the structure of the perco- 
lating cluster is geometrically built from weakly coordinated segments of ladders $(\theta \rightarrow 0)$, and weakly coordinated dimers $(\theta \rightarrow \pi / 2)$, e.g., finite necklace-like structures and finite randomly decorated chains (see Fig. 2). Both, antiferromagnetic Heisenberg ladders ${ }^{30}$ and decorated chains 31.32 .33 , have quantum-disordered ground states with a finite correlation length $\xi_{0}$. In antiferromagnetic ladders the ground state has a RVB nature ${ }^{34}$, and for the necklace it has a dimer-singlet nature 31.32 . When segments of such structures become part of a larger cluster, as it is the case in the inhomogeneous percolation model, they locally retain ground state properties similar to the thermodynamic limit if their length $l$ is large compared to the correlation length $\xi_{0}$. This represents the core mechanism of non-trivial enhancement of quantum fluctuations through bond dilution, leading to novel quantum disordered phases.

\section{NUMERICAL METHODS}

The purely geometric problem of inhomogeneous percolation is studied using a generalized version of a highly efficient classical Monte Carlo algorithm ${ }^{36}$. In turn, the quantum magnetic Hamiltonian Eq. (2) is investigated by Stochastic Series Expansion (SSE) QMC simulations based on the directed-loop algorithm ${ }^{35}$. The ground state properties are systematically probed by efficiently cooling the system down to its physical $T=0$ behavior via a successive doubling of the inverse temperature $\beta$ (Ref. 4 ). This approach is necessary since in two-dimensional (2D) diluted systems 2D correlations are often mediated by narrow quasi-one-dimensional links, such that the temperature scale at which these correlations set in is much lower than in the clean $2 \mathrm{D}$ case.

The scaling analysis of the quantum simulation results is carried out in two complementary ways ${ }^{4}$. In the first approach, the lattice size is fixed at $L \times L$, and the entire system is simulated, including percolating and nonpercolating finite clusters. In the second approach, a fundamental theoretical tool is used to ascertain the presence or absence of order in the system: we focus exclusively on the percolating cluster, i.e. discarding the finite clusters that cannot carry long-range order. On a finite $L \times L$ lattice with periodic boundary conditions the percolating cluster is identified as the largest cluster, winding around at least one of the two spatial dimensions. The advantage of this approach is that a single run of QMC simulation evaluates the observables on both the full lattice and the largest (percolating) cluster. At the same time the largest cluster has non-negligible size fluctuations (of order $L$ ), leading to a significant error in the QMC data. An alternative approach, eliminating these size fluctuations, is to grow the percolating cluster freely from an initial seed up to a fixed size $N_{c}$ but without any restriction of lattice boundaries. Starting from a single occupied bond, one visits the six neighboring bonds, activating them with probability $P_{D}\left(P_{L}\right)$ if they are $D(L)$ bonds, etc. The procedure is repeated until no new bonds are generated. The cluster geometry is accepted only if its final number of sites matches exactly the required $N_{c}$. A drawback of this procedure is that the cluster build-up becomes time consuming for large $N_{c}$ and away from the percolation threshold because of the large rejection rate.

In the full-lattice studies, $L$ values up to 64 were used. Within the $\beta$-doubling scheme, inverse temperatures as high as $\beta=256 L$ have proven to be necessary to observe the physical $T=0$ behavior. For the studies on fixed- $N_{c}$ clusters we considered $N_{c}$ values up to 2048 and inverse temperatures as high as $\beta=8 N_{c}$. For each lattice size and for each point $\boldsymbol{P}=\left(P_{D}, P_{L}\right), 10^{2}-10^{3}$ realizations of the diluted lattice/percolating cluster were generated independently to obtain disorder-averaged observables.

\section{MAGNETISM ON THE PERCOLATING CLUSTER}

In this section, QMC results are discussed which address the evolution of antiferromagnetic order on percolating clusters upon tuning the nature of bond dilution from homogeneous $(\theta=\pi / 4)$ to inhomogeneous $(\theta \rightarrow 0$, $\pi / 2)$. A fundamental observation 4 is that the presence or absence of magnetic order in a diluted system and the nature of the transition from order to disorder are determined by the magnetic behavior of the percolating cluster. In two dimensions, only one percolating cluster can exist in the system. We denote with $\left\langle M_{c}\right\rangle$ the disorder-averaged $(\langle\ldots\rangle)$ staggered magnetization per site of a percolating cluster, which is estimated by

$$
\left\langle M_{c}\right\rangle=\sqrt{\left\langle m_{c}^{2}\right\rangle}=\sqrt{\left\langle\frac{3}{N_{c}^{2}} \sum_{i j}^{(c)}(-1)^{i+j} S_{i}^{z} S_{j}^{z}\right\rangle} .
$$

Here the summation $\sum^{(c)}$ is restricted to sites contained in the percolating cluster, and $N_{c}$ is the total number of these sites. This is to be contrasted with the overall magnetization of the diluted lattice with $N=L^{2}$ sites,

$$
\langle M\rangle=\sqrt{\left\langle m^{2}\right\rangle}=\sqrt{\left\langle\frac{3}{N^{2}} \sum_{i j}(-1)^{i+j} S_{i}^{z} S_{j}^{z}\right\rangle} .
$$

Given that only the percolating cluster contributes to long-range order in the thermodynamic limit, one finds that for $N \rightarrow \infty$

$$
\left\langle m^{2}\right\rangle=\left\langle\frac{3}{N^{2}} \sum_{i j}^{(c)}(-1)^{i+j} S_{i}^{z} S_{j}^{z}\right\rangle=\left\langle\frac{N_{c}^{2}}{N^{2}} m_{c}^{2}\right\rangle .
$$

Moreover, exploiting the self-averaging property of the size distribution of the percolating cluster ${ }^{4}$, close to the percolation threshold one can use

$$
\langle M(\boldsymbol{P})\rangle \approx A\left|\boldsymbol{P}-\boldsymbol{P}_{c}^{(c l)}\right|^{\beta} M_{c}(\boldsymbol{P}),
$$



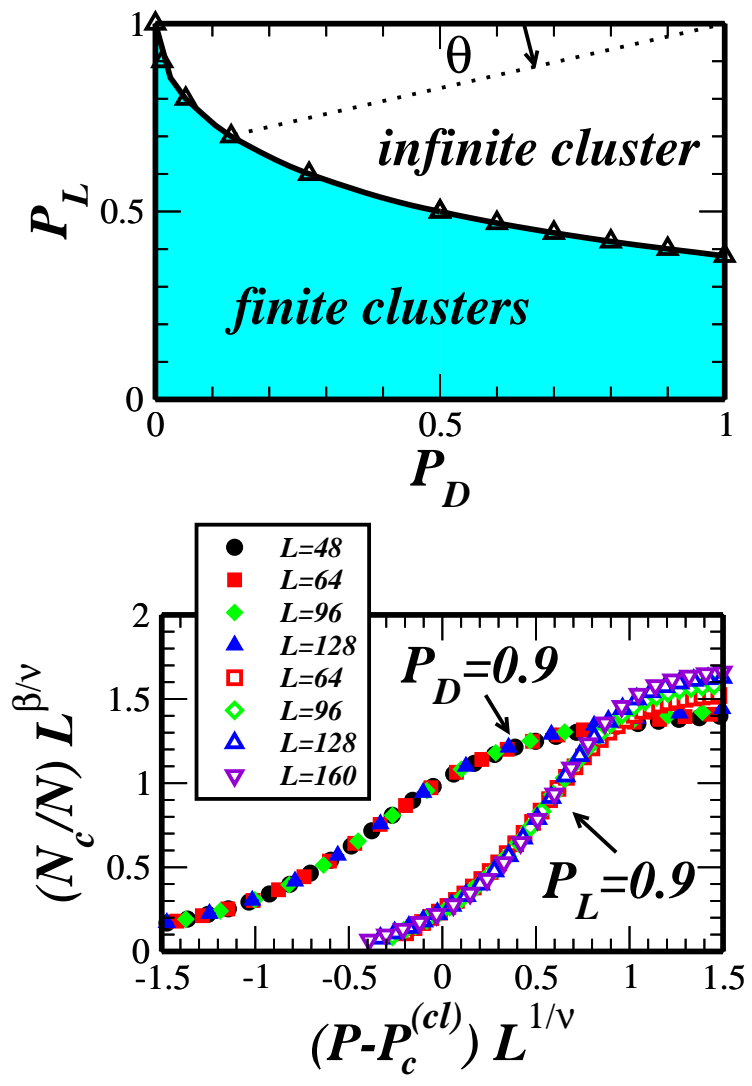

FIG. 3: (color online) Upper panel: classical phase diagram for the inhomogeneous bond-percolation problem on the square lattice. The angle $\theta$ parametrizing the critical curve is indicated. Lower panel: scaling plots for the network strength $N_{c} / N$ in the two strongly inhomogeneous cases $P_{D}=0.9$ and $P_{L}=0.9$. An excellent data collapse is realized, using the $2 \mathrm{D}$ percolation exponents $\beta=5 / 36$ and $\nu=4 / 3$, and with $P_{L, c}^{(c l)}=0.4007\left(P_{D}=0.9\right)$ and $P_{D, c}^{(c l)}=0.0107\left(P_{L}=0.9\right)$.

where $\boldsymbol{P}=\left(P_{D}, P_{L}\right)$ is the control parameter of the inhomogeneous percolation problem, and $\boldsymbol{P}=\boldsymbol{P}_{c}^{(c l)}$ defines the classical critical line of percolation thresholds in the $\left(P_{D}, P_{L}\right)$ space. In Eq. (6) we have used the critical behavior of the so-called network strength $\left\langle N_{c} / N\right\rangle \approx A\left|\boldsymbol{P}-\boldsymbol{P}_{c}^{(c l)}\right|^{\beta}$, where $A$ is a constant amplitude, and $\beta=5 / 36$ is the critical exponent of classical percolation 18 .

From Eq. (6) it is evident that, if $M_{c}\left(\boldsymbol{P}_{c}^{(c l)}\right) \neq 0$, the dilution-driven transition from magnetic order to disorder coincides with the classical percolation transition, both in terms of its location and of its critical exponents. Therefore only the absence of antiferromagnetic order on the percolating cluster can lead to a decoupling between the magnetic transition and the geometric percolation threshold.

The critical curve $\boldsymbol{P}=\boldsymbol{P}_{c}^{(c l)}$, determined from classical Monte Carlo simulations, is shown in Fig. 3 Despite the inhomogeneous nature of percolation for $P_{L} \neq P_{D}$,

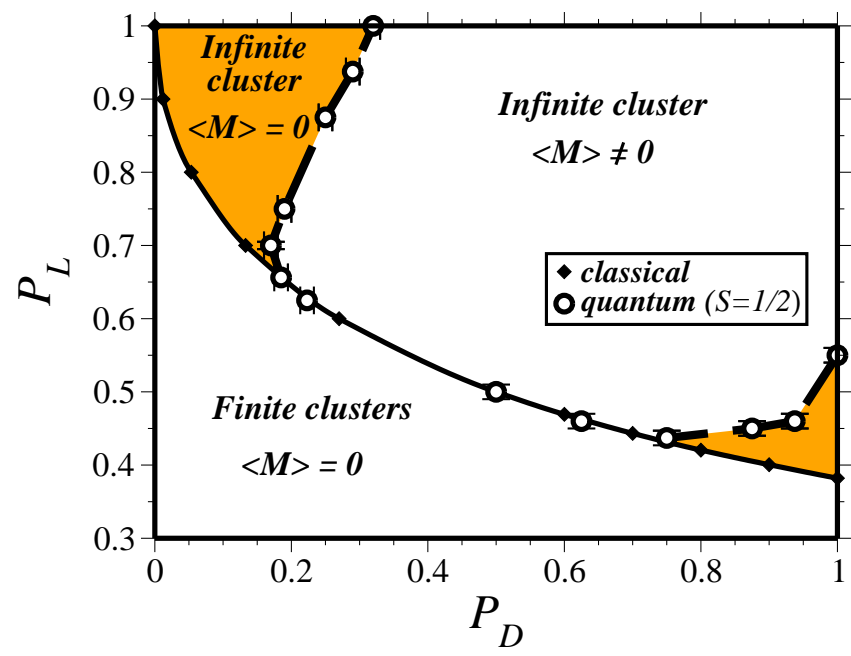

FIG. 4: (color online) Phase diagram of the spin-1/2 QHAF on the inhomogeneously bond-diluted square lattice.

excellent scaling of the classical MC results is observed using the $2 \mathrm{D}$ percolation exponents ${ }^{18}$. This demonstrates that the universality class remains unchanged along the critical curve.

Starting from the classical Monte Carlo result, the evolution of the order parameter can be tracked on the percolating cluster $\left\langle M_{c}\left(\boldsymbol{P}_{c}^{(c l)}\right)\right\rangle$ along the critical line $\boldsymbol{P}=\boldsymbol{P}_{c}^{(c l)}$ to monitor the effect of quantum fluctuations enhanced by inhomogeneity. To this end, we perform a scaling study of the staggered magnetization on percolating clusters for fixed size $N_{c}$, with $N_{c}$ up to 2048 and $\beta=8 N_{c}$. The obtained data are extrapolated to the thermodynamic limit by a three-parameter polynomial fit $\left\langle m_{c}^{2}\left(N_{c}\right)\right\rangle=\left\langle m_{c}^{2}(\infty)\right\rangle+a N_{c}^{-1 / 2}+b N_{c}$.

At the homogeneous bond percolation threshold $P_{D}=$ $P_{L}=0.5$ the percolating cluster is found to have antiferromagnetic long-range order, in agreement with Ref. 4. However, the finite value of the order parameter is strongly reduced as the inhomogeneity is turned on, both in the "dimer" $(\theta \rightarrow \pi / 2)$ and the "ladder" $(\theta \rightarrow 0)$ directions, until it vanishes at non-trivial $\theta$ values, $\theta_{L} \approx$ 0.34 in the ladder limit and $\theta_{D} \approx 1.23$ in the dimer limit. This means that inhomogeneous percolation exhibits non-linear quantum fluctuations that are able to destroy the antiferromagnetic long-range order, in contrast to the homogeneous percolation case ${ }^{4}$ with a renormalized classical ground state.

According to Eq. (6) this must be reflected in a profound change of the critical properties, since the vanishing of the staggered magnetization on the percolating cluster decouples the magnetic transition from the percolation threshold. Beyond the two critical values $\theta_{L}$ and $\theta_{D}$, a higher concentration of bonds will be required for the system to magnetically order than for the lattice to percolate, thus opening up an intermediate phase with a novel quantum-disordered ground state. 

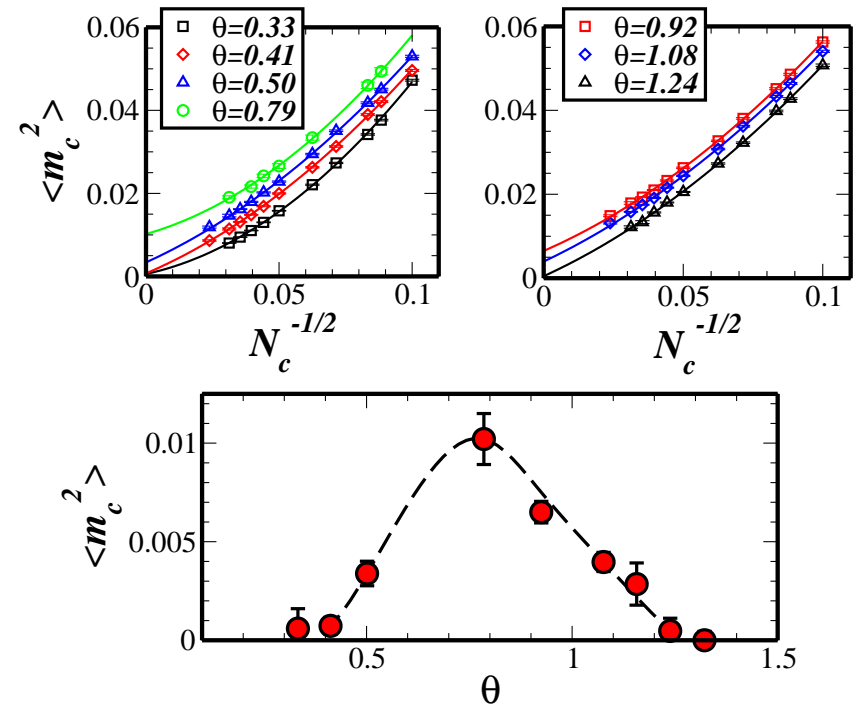

FIG. 5: (color online) Upper panel: scaling of the disorderaveraged squared staggered magnetization on the percolating cluster with fixed size $N_{c}$. The different curves correspond to various points along the classical percolation transition. The continuous lines represent quadratic fits. Lower panel: extrapolated thermodynamic values of the staggered magnetization from the upper panel as a function of the inhomogeneity parameter $\theta$. The dashed line is a guide to the eye.

\section{PHASE DIAGRAM}

In this section, QMC results are discussed for the magnetic phase diagram in the $\left(P_{D}, P_{L}\right)$ plane. The magnetic transitions are located via scaling of the spin-spin correlation length $\xi$, extracted from the structure factor through the second moment estimator ${ }^{37}$. The inhomogeneity of bond dilution breaks the discrete rotation symmetry of the lattice, such that two distinct correlation lengths $\xi_{x}$ and $\xi_{y}$ need to be considered along the $x$ and $y$ lattice directions. At critical points separating 2D long-range order from disordered phases, both correlation lengths must scale linearly with the lattice size, $\xi_{x}, \xi_{y} \sim L$, regardless of the universality class of the transition. In locating the magnetic transitions we have verified that this condition is satisfied. This leads to the conclusion that, despite its inhomogeneity, the magnetic system has unique well-defined phase transitions.

The resulting phase diagram is shown in Fig. 4 The magnetic transition line coincides with the percolation line for moderate inhomogeneity. As discussed in the previous section, in this region of the phase diagram the percolating cluster at the percolation threshold is antiferromagnetically ordered. Therefore not only the magnetic transition and the geometric transition coincide, but also the critical exponents of the magnetic transition are those of 2D percolation 23 , according to Eq. (6).

For strong inhomogeneity. the scenario of a classical percolation transition in the magnetic Hamiltonian is precluded by non-linear quantum fluctuations. In fact, two multicritical points occur, in the ladder and in the dimer direction, beyond which the magnetic transition decouples from the percolation threshold. The location of these multicritical points is quantitatively consistent with the vanishing of antiferromagnetic order on the percolating cluster at two critical values of the inhomogeneity parameter $\theta$ (Fig. [5).

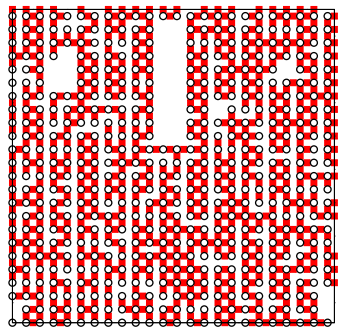

(a)

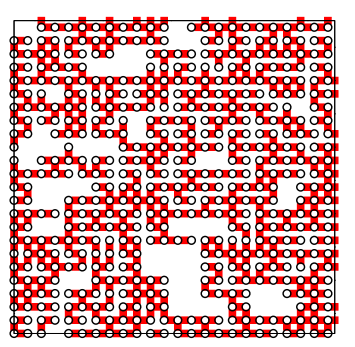

(c)

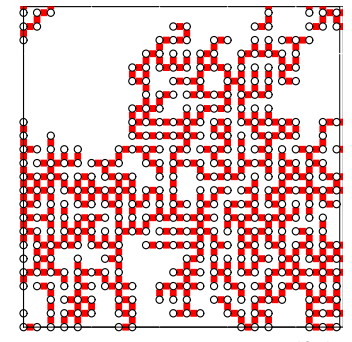

(b)

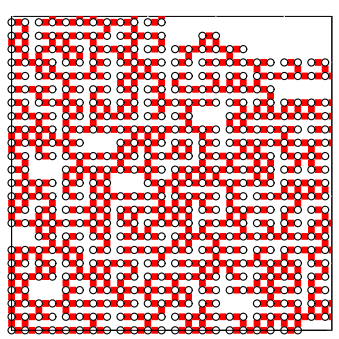

(d)
FIG. 6: (color online) Percolating clusters for the bonddiluted square lattice with size $24 \times 24$ and periodic boundary conditions: (a) $P_{D}=0.15, P_{L}=0.8$; (b) $P_{D}=0.5, P_{L}=0.5$ (homogeneous case); (c) $P_{D}=0.9, P_{L}=0.45$; (d) $P_{D}=1.0$, $P_{L}=0.44$ (randomly coupled dimers).

Beyond the two multicritical points, intermediate quantum phases appear, in which antiferromagnetic order is absent on the percolating cluster both at and away from the percolation threshold. These regions represent novel quantum-disordered phases on an infinite family of $2 \mathrm{D}$ percolated random lattices. Representative structures of this family are shown in Fig. [6 together with the homogeneous percolating cluster. It is remarkable that the homogeneous percolating cluster (Fig. 6(b)) has long-range antiferromagnetic order in the thermodynamic limit, whereas the other percolating clusters in Fig. 6(a),(c),(d) do not. In fact, the latter structures have a significantly higher average number of bonds per site than the homogeneous percolating cluster, which has a fractal dimension $\frac{18}{18}$ lower than $2\left(D_{f}=91 / 48\right)$. A detailed discussion of the nature of the quantum-disordered phase will be given in Sec. VII]

The evolution of the critical exponents upon tuning inhomogeneity has been investigated in Ref. 23. Surprisingly, the two multicritical points do not mark an evident discontinuity in the universality class of the model, and the classical percolation exponents appear to persist 
also for intermediate inhomogeneity beyond the multicritical points, changing to radically different values only in the extreme inhomogeneous limits. Therefore the magnetic quantum phase transitions close to the multicritical points appear to retain a percolative character. This result can be understood within the following argument. To destroy the long-range nature of a random network it is sufficient to cut a few links on its backbone. This implies that the non-linear quantum fluctuations leading to quantum-disorder on the percolating cluster need only have short-wavelength components, in contrast to conventional quantum phase transitions in translationally invariant lattices. Indeed, strongly fluctuating states appearing on links made of segmented ladders or decorated chains (see Sec. III) have a markedly local effect on magnetic correlations, i.e. they magnetically decouple the portions of the percolating cluster connected through that link. This effect is equivalent to geometrically removing that link, thus leaving two portions of the cluster disconnected $^{23}$. If weak links occur on the backbone of the percolating cluster, this mechanism is sufficient to lead to quantum disorder. Only quantum fluctuations with wavelengths comparable to the length of the weak link are required. To reestablish magnetic correlations between the two portions of the percolating cluster disconnected by quantum fluctuations, it is then necessary to add more bonds to the cluster in order to find an alternative path for magnetic correlations to spread over the cluster. Since this process is of geometrical character it endows the quantum phase transition with a percolative nature.

The above picture of a percolative quantum phase transition breaks down for strong inhomogeneity. In this limit, the building blocks of the percolating cluster are the strongly quantum fluctuating substructures depicted in Fig. 2] and hence the quantum phase transition is driven by the competition between the energy scale of the gap above the ground state of such substructures and the energy scale of the spatially random couplings between the substructures. In a real-space picture, the competition occurs between the finite correlation length of the substructures and the characteristic length set by the spacing between the spatially random couplings. This competition will be discussed in detail in the next section. The quantum phase transition is completely disconnected from classical percolation of the lattice, and it is reasonable to expect for it to be in a different universality class, as concluded in Ref. 23.

\section{CRITICALITY NEAR THE LADDER LIMIT}

So far, the onset of the quantum disordered phases was discussed, approaching them from the homogeneous limit of Eq. (11). A complementary understanding of the novel quantum phase and the associated quantum phase transitions can be gained by starting from the extreme inhomogeneous limit of randomly coupled ladders $(\theta=0)$.
This limit lends itself to a very simple analysis in terms of the properties of the single spin- $1 / 2$ antiferromagnetic Heisenberg ladder.

In the limit of $P_{L}=1$, the system is composed of an array of two-leg ladders randomly coupled by $D$ bonds. The probability of activation of a dimer bond is reflected in the average distance $\langle r\rangle$ of two $D$ bonds along a column separating two neighboring ladders, namely $P_{D}=1 /\langle r\rangle$. A single $D$ bond is sufficient to connect two ladders geometrically. In other words, $\langle r\rangle=L$ is sufficient to connect all ladder subsystems on a $L \times L$ lattice. Taking the limit $L \rightarrow \infty$, one obtains immediately $P_{D} \rightarrow 0$. Thus geometrically the system percolates at any infinitesimal concentration $P_{D}>0$ and the percolating cluster is the entire lattice itself.

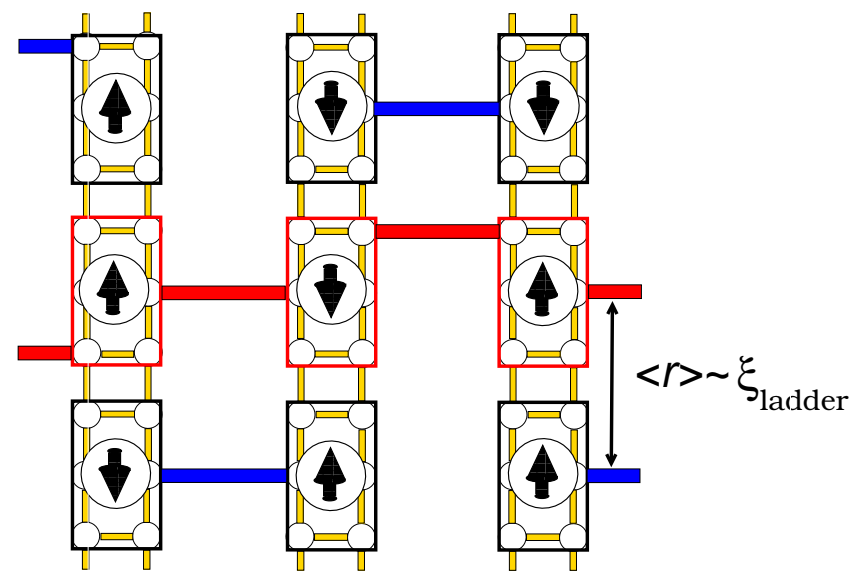

FIG. 7: (color online) Cartoon of the ordering mechanism in randomly coupled ladders. When $P_{D}=1 /\langle r\rangle \approx 1 / \xi_{\text {ladder }}$ (see text) percolating strings of block spins (rectangles) appear in the system. The divergence of the correlation length along such strings drives the onset of long-range correlations between the strings and ultimately of $2 \mathrm{D}$ long-range order.

However the system does not develop antiferromagnetic order until a finite value $P_{D} \approx 0.32$ is reached. This is not surprising since each two-leg ladder has a quantum-disordered ground state with a finite excitation gap. This gap has to be overcome before the Néel ordered state can become the ground state. A more quantitative argument can be formulated based on the fact that each isolated ladder has a finite correlation length ${ }^{34}$ $\xi_{\text {ladder }} \approx 3.19$. One can imagine a renormalization group transformation that creates effective block spins from all the spins within a correlation volume in such a way that all block spins are perfectly uncorrelated with each other (see Fig. (7). When turning on the $D$ bonds, it becomes evident that, to establish long-range correlations in the system, it is necessary to have a string of block spins percolating from one side to the other of the system. Given that all block spins are independent within the same ladder, this is only possible if, on average, each block spin is connected by a $D$ bond to both its right and left neigh- 
boring block spin. This requires an average spacing of the $D$ bonds of $\langle r\rangle \approx \xi_{\text {ladder }}$ on each column, which in turn leads to the following estimate of the critical concentration of dimer bonds

$$
P_{D, c}=\frac{1}{\xi_{\text {ladder }}} .
$$

Despite the simplicity of the argument, this estimate is surprisingly good: $P_{D, c} \approx 0.313$ to be compared with the QMC result $P_{D, c}=0.32(1)$.

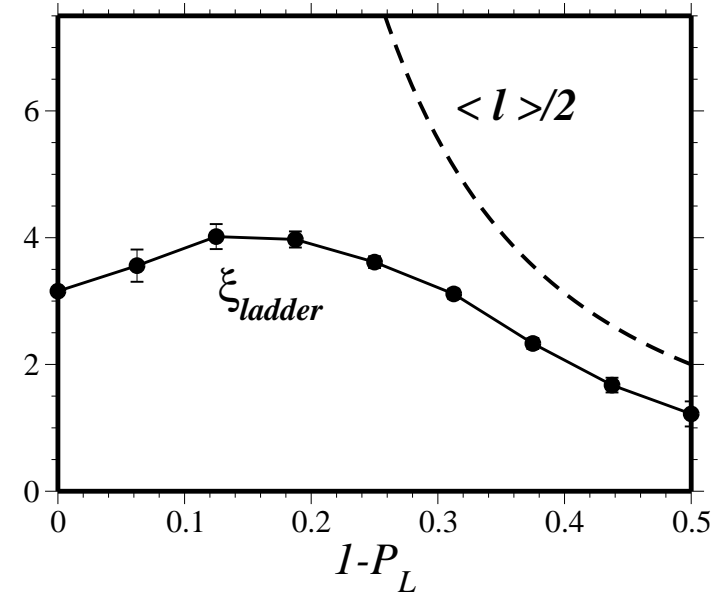

FIG. 8: Correlation length of the bond-diluted $128 \times 2$ ladder compared with the average ladder length $\langle l\rangle$. The data for $\xi_{\text {ladder }}$ are taken at $\beta=2048$; comparison with data at $\beta=$ 4096 (not reported) shows no significant deviation.

Interestingly, this argument can be extended to the case of randomly coupled weakly diluted ladders, namely for $P_{L} \lesssim 1$. The correlation length of the ladder remains the dominant length scale of the problem, as long as it is much smaller than the new length scale introduced by the dilution

$$
\langle l\rangle=\frac{1}{\left(1-P_{L}\right)^{2}},
$$

corresponding to the average length of the ladder segments after dilution.

To test to which extent the above argument is applicable we performed SSE-QMC simulations on a single bond-diluted two-leg ladder to determine the evolution of the correlation length upon dilution. The results are shown in Fig. 8. For low doping concentrations, we observe that the average correlation length of the ladder increases moderately. For higher concentrations of missing bonds, the average ladder length $\langle l\rangle$ becomes comparable to the correlation length, which then crosses over to a decreasing behavior simply reflecting that of $\langle l\rangle$.

We now focus on the increasing tendency for small dilution. This behavior reflects various competing mechanisms. The dominant effects having the highest probabilities $\left(\mathcal{O}\left(P_{L}\right)\right.$ and $\left.\mathcal{O}\left(P_{L}^{2}\right)\right)$ are:

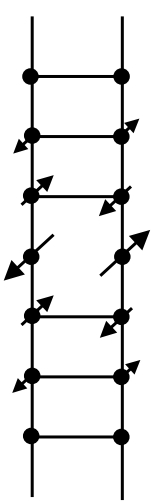

(a)

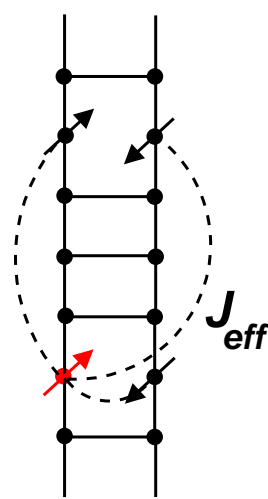

(b)

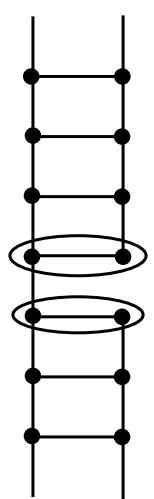

(c)
FIG. 9: (color online) Dominant effects of bond dilution on a two-leg ladder: (a) local antiferromagnetic modulation due to a missing rung bond; (b) effective couplings between spins missing their rung bond; (c) enhancement of the singlet component (indicated by an ellipse) of the two rung dimers adjacent to a missing leg bond.

(a) The removal of a rung bond results locally in two uncoupled $S=1 / 2$ free moments which introduce a local staggered modulation of the adjacent spins $\frac{38,39}{}$ within a correlation volume $\sim \xi_{\text {ladder }}$ (Fig. 9(a)). This leads to a local increase in the antiferromagnetic correlations along the legs;

(b) If two rung bonds are removed sufficiently close to each other, the induced local antiferromagnetic modulations of the ladder ground state "lock in phase", leading to an effective coupling between the free moments, exponentially decaying with the distance in the limit of low doping 39.40 ,

$$
J_{\text {eff }} \sim(-1)^{i-j} \exp \left[-|i-j| / \xi_{\text {ladder }}\right] .
$$

These long-range interactions also lead to an increase of the correlation length in the system through an order-by-disorder mechanism (Fig. 9(b));

(c) The removal of one leg bond, on the contrary, leads to a local enhancement of the rung-singlet component of the state of the two adjacent rungs (Fig. 9(c)). This mechanism weakens the correlations along the two legs.

Effect (c) has twice the probability compared to the effects (a) and (b) because there are twice as many leg bonds as rung bonds. The non-trivial competition between correlation enhancement (a)-(b) and correlation suppression (c) has the combined effect that in bonddiluted ladders, the correlation length always remains $f$ nite down to zero temperature. This is to be contrasted with the case of site-diluted ladders, in which the correlation length diverges algebraically with decreasing temperature, $\xi \sim T^{-0.4}$, as reported in Ref. 41. (Obviously, even in the site-diluted case the average correlation length is eventually upper-bounded by the average length of the ladder segments). It is interesting to note 
that for the case of site dilution, correlation-suppressing effects are not present beside the simple fragmentation of the ladder.

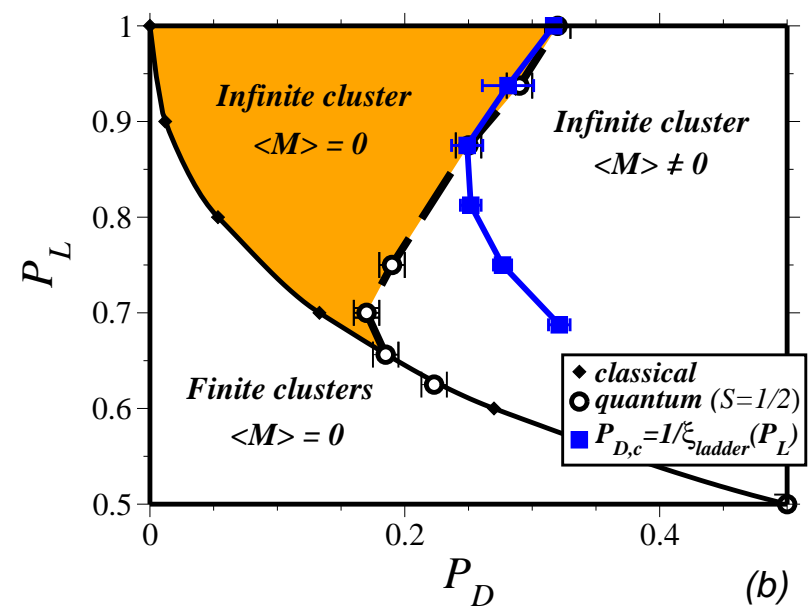

FIG. 10: (color online) Phase diagram of the QHAF on the inhomogeneously bond-diluted square lattice (see also Fig. (4) close to the ladder limit and compared with the single-ladder prediction $P_{D, c}=1 / \xi_{\text {ladder }}\left(P_{L}\right)$ (see text).

The absence of diverging correlations in bond-diluted ladders is crucial for the occurrence of an extended quantum-disordered phase. The finite correlation length of a single ladder for $P_{L} \lesssim 1$ allows us to repeat the RG argument sketched in Fig. 7 for the case of bond-diluted ladders. One needs a finite and non-classical concentration of dimer bonds $P_{D, c}$ to drive the system into a Néel ordered phase, directly related to the quantum correla-

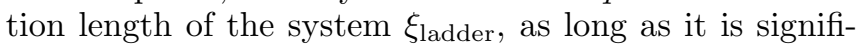
cantly lower than the classical length $\langle l\rangle$ :

$$
P_{D, c}\left(P_{L}\right) \approx \frac{1}{\xi_{\text {ladder }}\left(P_{L}\right)} .
$$

The above estimate is a theoretical prediction for the quantum-critical curve $\boldsymbol{P}_{c}=\left(P_{D, c}, P_{L, c}\right)$, which can be directly compared with the QMC results, shown in Fig. 10. The range of quantitative validity of Eq. 10] is surprisingly large, extending down to $P_{L} \approx 0.875$, which is interestingly also the value where the quantum correlation length $\xi_{\text {ladder }}\left(P_{L}\right)$ starts to cross over towards the decreasing classical behavior (Fig. 8). At this point the ground state of the ladder segments is strongly altered by the presence of the dimer bonds, and hence the argument leading to Eq. (10) breaks down.

\section{THE QUANTUM-DISORDERED PHASE: CORRELATIONS AND GRIFFITHS-MCCOY SINGULARITIES}

In this section, we discuss the nature of the quantumdisordered phase. Ground-state properties and thermo- dynamic observables are considered which reveal the nature of the low-lying excitation spectrum. A fundamental aspect of this phase, as it is typical for disordered systems, are large variations in the local properties of the system, reflecting the local geometric structure defined by disorder. In the previous sections, we have discussed how the presence of segments of ladders or decorated chains leads to the local formation of RVB/dimer-singlet states. At the same time, bond dilution of ladders leads to the appearence of $S=1 / 2$ local degrees of freedom, effectively interacting across the strongly quantum fluctuating regions. The coexistence of such different subsets of spins within the same system raises the question of what the global nature of the ground state really is.

\section{A. Short-range correlations}

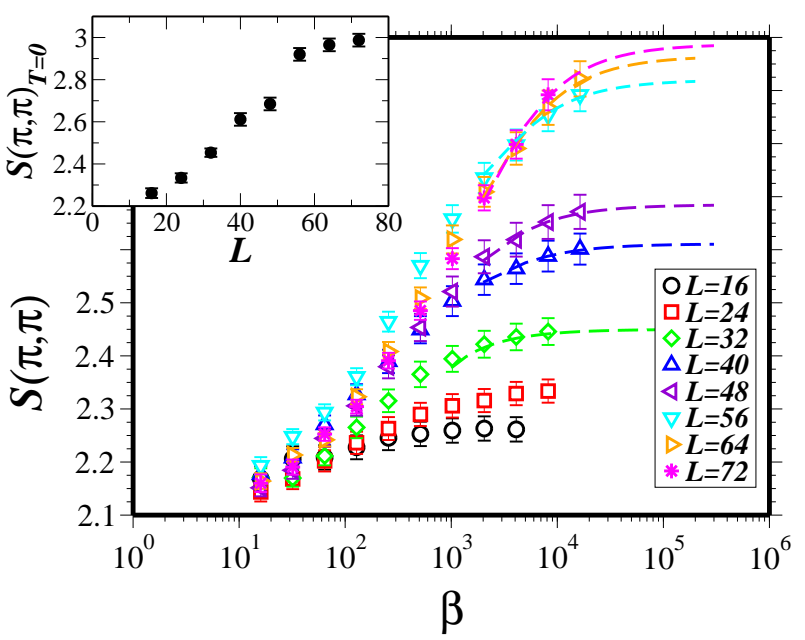

FIG. 11: (color online) Temperature and finite-size scaling of the static structure factor $S(\pi, \pi)$ for a representative point in the quantum-disordered regime $\left(P_{D}=0.15, P_{L}=0.85\right)$. Dashed lines are a quartic polynomial fit in $T=\beta^{-1}$ [see Eq. [11] to extrapolate to the $\beta \rightarrow \infty$ limit (when required). Inset: $\beta \rightarrow \infty$ extrapolated values of the static structure factor plotted vs. system size, clearly showing saturation in the thermodynamic limit.

We first characterize the ground state in terms of global correlation properties. The absence of magnetic order in this phase has already been discussed in Sec. IV] Fig. 11 shows the staggered structure factor $S(\pi, \pi)$ for a representative point $\left(P_{D}=0.15, P_{D}=0.85\right)$ in the quantum disordered phase on the "ladder" side of the phase diagram in Fig. 4 The finite-temperature data for $S(\pi, \pi)$, obtained using $\beta$-doubling, are extrapolated to the limit $T \rightarrow 0$ by accounting for power-law temperature corrections up to fourth order:

$$
S(\pi, \pi ; T)=S(\pi, \pi)_{T=0}+\sum_{i=1}^{4} \frac{a_{i}}{\beta^{i}}
$$




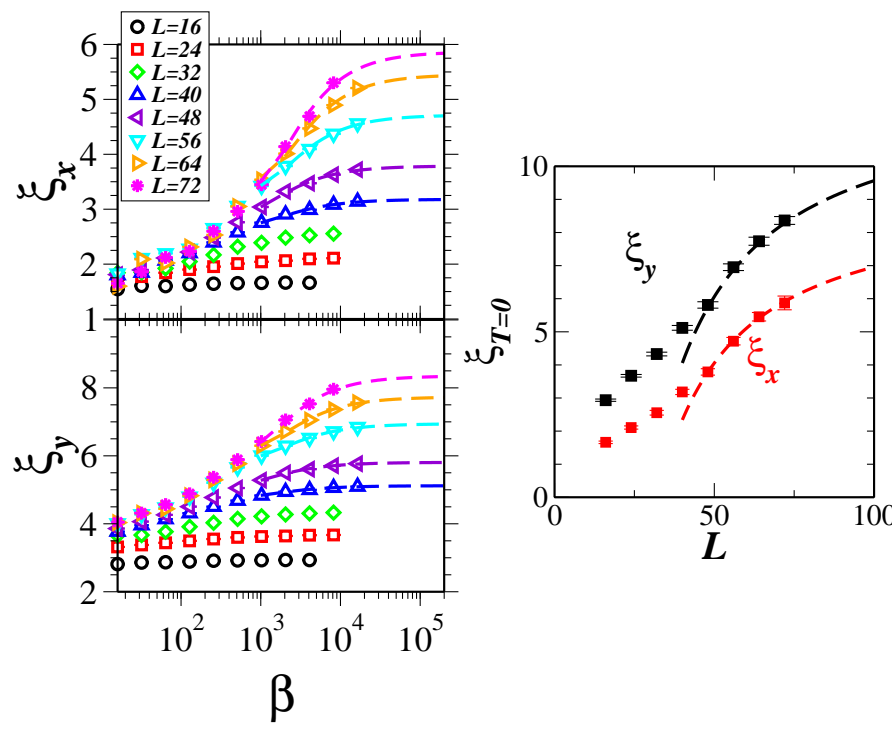

FIG. 12: (color online) Left panel: temperature and finitesize scaling of the correlation length along the two lattice directions $\left(\xi_{x}\right.$ and $\left.\xi_{y}\right)$ for a representative point in the quantumdisordered regime $\left(P_{D}=0.15, P_{L}=0.85\right)$. Dashed lines are a quartic polynomial fit in $T=\beta^{-1}$ to extrapolate to $\beta \rightarrow \infty$ (when required). Right panel: $\beta \rightarrow \infty$ extrapolated values of the correlation length(s) vs. system size; the dashed lines are polynomial fits up to second order in $L^{-1}$ on the points for $L \geq 48$ to compensate for the finite-size effects.

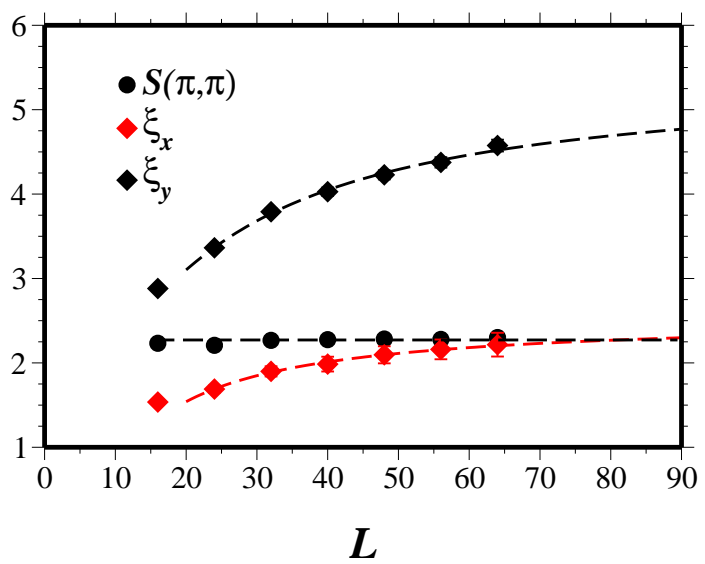

FIG. 13: (color online) Finite-size scaling of the $T=0$ static structure factor and correlation length(s) for a point in the quantum-disordered regime $\left(P_{D}=0.15, P_{L}=0.9\right)$ closer to the ladder limit. The dashed lines associated with the correlation length data are quadratic fits $\xi(L)=\xi(\infty)+a / L+$ $b / L^{2}$.

where the $a_{i}$ 's are fitting coefficients. The extrapolated $T=0$ values of $S(\pi, \pi)$ are shown in the inset of Fig. 11] as a function of system size. They clearly display saturation for $L \rightarrow \infty$, proving not only that the system is disordered, but also that it has finite-range correlations.

This fact is further reflected in the correlation length $\xi_{x(y)}$ along the two lattice dimensions, shown in Fig. 12 A fitting procedure similar to Eq. (11) is used to eliminate polynomial finite-temperature correction. The $\xi_{T=0}$ values so obtained (right panel of Fig. 12) suggest convergence towards a finite value for $L \rightarrow \infty$, although the correlation length is large for the particular point $\left(P_{D}=0.15, P_{D}=0.85\right)$, and, even for the largest considered size $(L=72), \xi$ does not display full saturation. A polynomial fit $\xi(L)=\xi_{\infty}+a / L+b / L^{2}$ for $L \geq 48$ yields saturation values $\xi_{x, \infty}=9(1)$ and $\xi_{y, \infty}=12.5(5)$, resulting in a considerable correlation volume of $\sim 100$ sites. It is important to keep in mind that the finite correlation length in the quantum disordered system varies strongly with the degree of inhomogeneity, and it can become as small as $\xi \sim 3$ in the limit of randomly coupled ladders. For instance, Fig. 13] shows the finite-size scaling of the static structure factor and correlation lengths for a point closer to the ladder limit, $P_{D}=0.15$ and $P_{L}=0.9$. For this point one observes excellent temperature saturation of the data up to $L=64$ for $\beta \leq 16384$ without the need of any fitting procedure. Moreover, the zero-temperature data reported in Fig. 13 show a much better saturating behavior within the system sizes considered, leading to the asymptotic values $\xi_{x, \infty}=2.5(1)$ and $\xi_{y, \infty}=5.4(2)$.

A clear picture of the short-range nature of correlations also emerges from real-space images of

- the local staggered structure factor,

$$
S(\pi, \pi ; i)=\sum_{j \neq i}(-1)^{i+j}\left\langle S_{i}^{z} S_{j}^{z}\right\rangle_{0} ;
$$

- the correlation function $C(0, i)=\left\langle S_{0}^{z} S_{i}^{z}\right\rangle_{0}$ between a reference site and all the other spins;

- the energy of each bond $E_{\langle i j\rangle}=J\left\langle\boldsymbol{S}_{i} \cdot \boldsymbol{S}_{j}\right\rangle_{0}$,

all shown in Fig. 14 for a given sample corresponding to a representative point of the quantum-disordered phase on the "ladder side". Here the symbol $\langle\ldots\rangle_{0}$ indicates the expectation value associated with the ground state of the specific sample considered, not to be confused with the disorder average $\langle\ldots\rangle$.

Starting with the bond energy, we observe that quantum fluctuations introduce a wide range of variability in its values, energetically promoting weakly correlated dimers or quadrumers and rung bonds on ladder segments, at the expenses of the energy of the adjacent bonds (due to the fact that a $S=1 / 2$ particle can form a singlet with only one other $S=1 / 2$ particle). In particular the rare $D$ bonds have in general the weakest energies, due to the strong tendency of the energy to be minimized on the ladder segments. This leads to a clear quantum suppression of correlations in the direction transverse to the ladders.

The local staggered structure factor of Eq. (12) measures the "effective correlation volume" (integral of the antiferromagnetic correlation function) around each spin, thus identifying the spins that are most strongly correlated with the remainder of the system. In Fig. 14(a) 


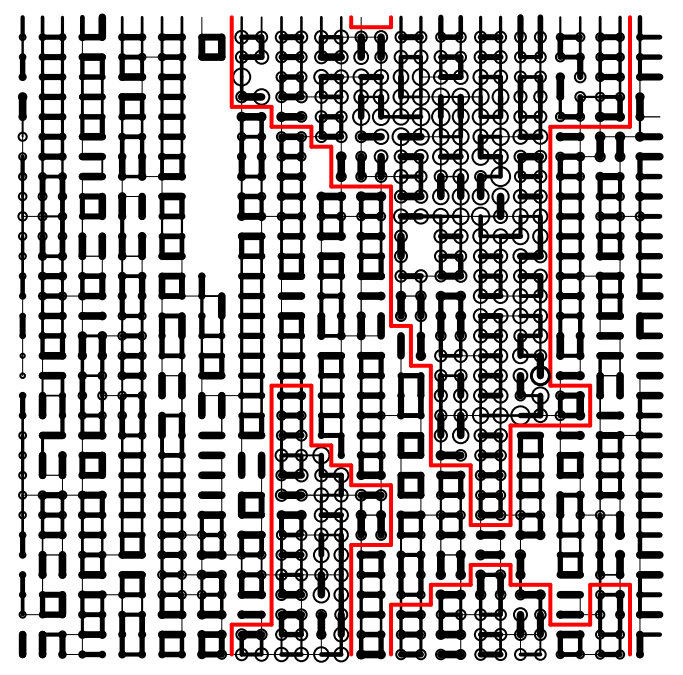

$$
\begin{array}{ll}
\mathrm{E}=-\mathrm{J} / 2 & -\mathrm{E}=-\mathrm{J} / 4 \\
\mathrm{~S}(\mathrm{i})=2.5 &
\end{array}
$$

(a)

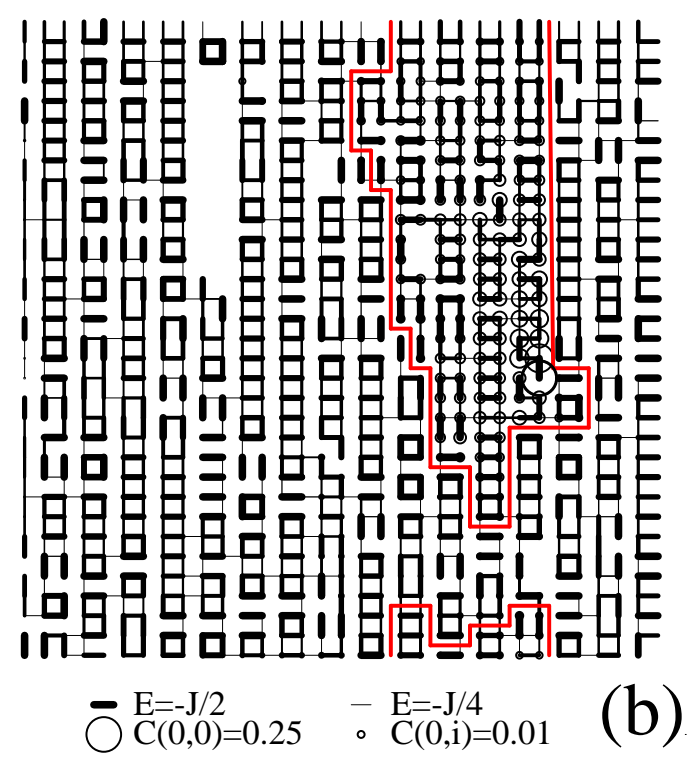

FIG. 14: (color online) Real-space images of the bond energy (both panels), local static staggered structure factor $S(i)$ (see text) (a) and of the static staggered correlations $C(0, i)$ between a reference point on a dangling bond and the remainder of the system (b), for the percolating cluster on a $32 \times 32$ sample with $P_{D}=0.15$ and $P_{L}=0.85$ at $\beta=8192$. The thickness of each bond is proportional to its energy, whereas the radius of the dots in (a) and (b) is proportional to the local value of $S(i)$ and $C(0, i)$. In (a) red boundaries highlight the regions where local correlations exceed the average value, namely where $S(i) \gtrsim S(\pi, \pi)$. In (b) the red boundaries mark the correlation volume beyond which $C(0, i) \leq 8 \times 10^{-3}$, corresponding to a distance (in units of the correlation length) $\left|r_{i}\right| / \xi \approx \ln [C(0,0) / C(0, i)] \approx 3.4$.

we observe that only a portion of the spins has a sizable local structure factor, when they belong to regions with a larger local coordination number. In Fig. 14(b) we have picked a site on a dangling bond attached to a segment of a ladder, and calculated its correlations to the rest of the system. Compared with Fig. 14(a), we observe that the correlation volume around the chosen spin is considerably smaller than the cluster of "correlated" spins identified by the local structure factor. This implies that spins that are most correlated with the remainder of the system are not all correlated with each other. The finite-range nature of correlations is also clearly shown in the picture, along with the non-monotonic decay of correlations with distance. The latter is due to the fact that a dangling spin is mostly correlated with spins not involved in a local singlet state, even over long distances, due to the effective long-range couplings discussed in Sec. [VI Nonetheless, even in the presence of such couplings, correlations decay significantly with distance. This picture is to be contrasted with the case in which long-range couplings give rise to a genuine order-by-disorder phenomenon, as for instance in the site-diluted dimerized systems treated in Ref. 42. There the real-space image of $C(0, i)$ shows very little spatial decay of the correlations between the free moments of the system.

\section{B. Griffiths-McCoy singularities}

In this section, the nature of the low-lying excitation spectrum in the quantum-disordered phase is discussed. In a clean system, the presence of a finite real-space correlation length implies finite correlations also in imaginary time, i.e. the existence of a gap in the excitation spectrum. In disordered systems, however, this is not necessarily the case. The disorder considered here is completely uncorrelated in real space but perfectly correlated in imaginary time, such that long-range correlations in the time dimension (and thus absence of a gap) may coexist with short-range real-space correlations.

The nature of the low-lying excitation spectrum is reflected in the low- $T$ uniform susceptibility. The global uniform susceptibility of the system is typically dominated by the Curie contribution from odd-numbered finite clusters, which masks the behavior of the quantumdisordered percolating cluster. Hence, in the following discussion the contributions from the finite clusters are neglected. Instead, we focus on the uniform susceptibility of the percolating cluster with an even number of sites $N_{c}$ :

$$
\chi_{u, c}=\frac{\beta}{N_{c}} \sum_{i j \in c}\left\langle S_{i}^{z} S_{j}^{z}\right\rangle
$$

The results for $\chi_{u, c}$ as a function of temperature, both in the ladder regime and in the dimer regime, are shown in Fig. 15. A gapped spectrum would imply an exponentially vanishing susceptibility as $T \rightarrow 0$. However, we find a non-vanishing susceptibility in this limit, and observe that $\chi_{u, c}$ instead displays a power-law divergence 

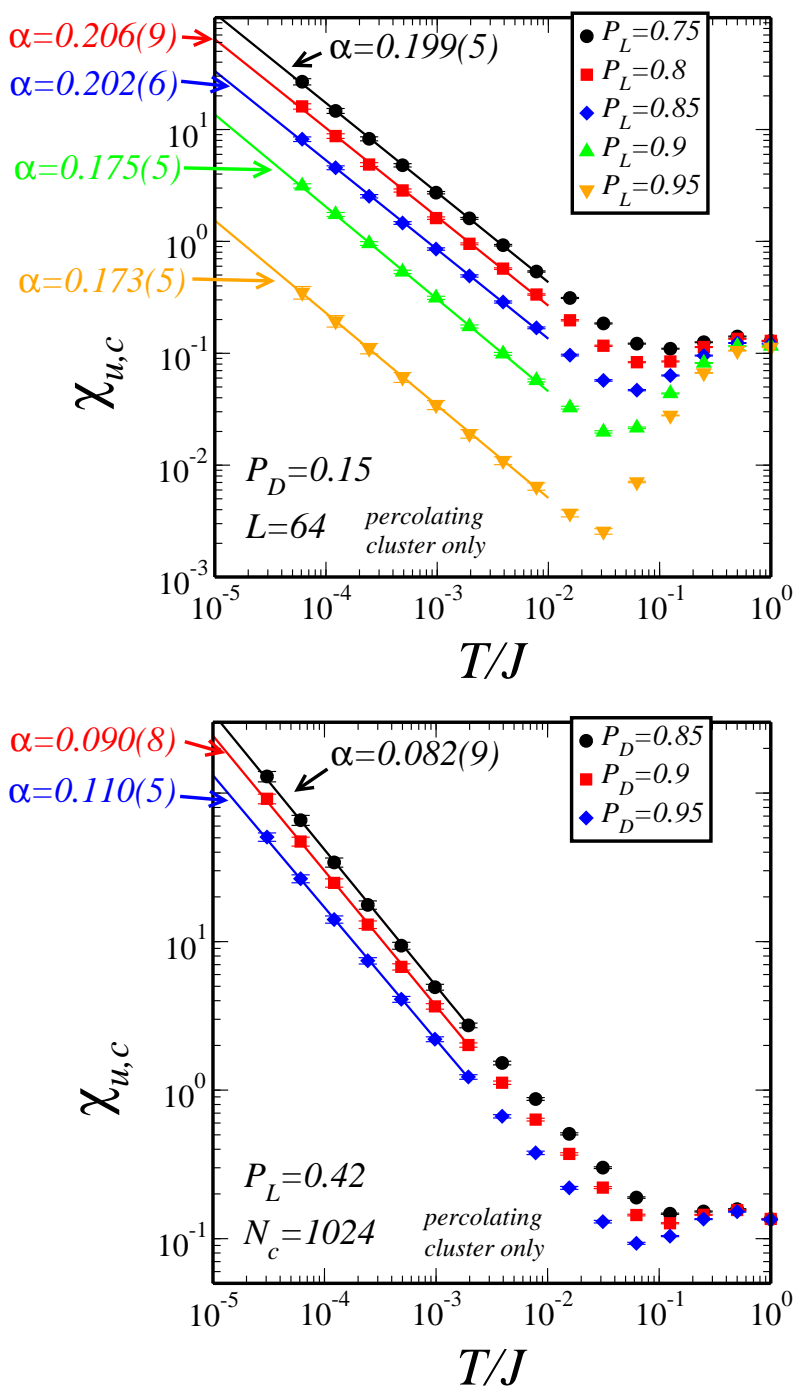

FIG. 15: (color online) Low-temperature uniform susceptibility of the percolating cluster in the quantum-disordered regime: ladder regime (upper panel) and dimer regime (lower panel). The solid lines are power-law fits of the form $\chi_{u, c} \sim$ $T^{-1+\alpha}$, and the resulting fit coefficients $\alpha$ are indicated. In the upper panel, the percolating cluster is picked as the largest cluster in a $64 \times 64$ lattice, whereas in the lower panel it is grown freely from a seed site up to a fixed site of $N_{c}=1024$ (see Sec. III).

$$
\chi_{u, c} \sim T^{\alpha-1}
$$

with $\alpha \neq 0$, such that this divergence is not Curie-like. In particular, $\alpha=\alpha(\boldsymbol{P})$ varies continuously as one scans across the quantum-disordered region: $\alpha \approx 0.09 \div 0.2$. This clearly shows that the system is not only gapless, but that its gapless excitations lead to singularities in the response to an external field.

Quantum-disordered phases with divergent response properties at $T \rightarrow 0$ are familiar in the literature of disordered systems. They are classified as quantum Griffiths phases, and have been theoretically shown to exist in bond-disordered dimerized chains ${ }^{7}$, in bonddisordered $S=1$ (Haldane) chains ${ }^{43.44}$, and in disordered transverse-field Ising models ${ }^{10.11 .12}$ (see Ref. 45 for a recent review). As in our model, their central feature is the coexistence of short-range correlations together with socalled Griffiths-McCoy singularities in the response functions. Such singularities are due to the anomalous role played by local low-energy excitations living on rare regions of the system.

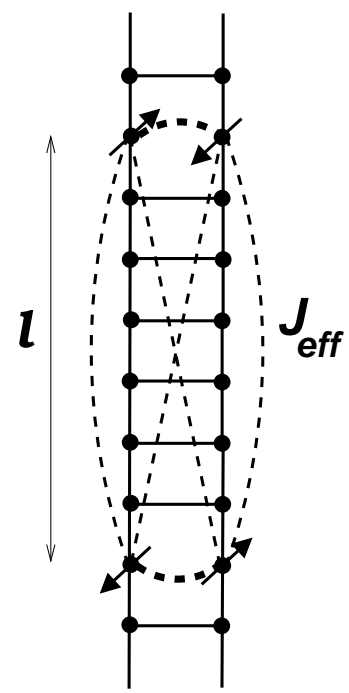

(a)

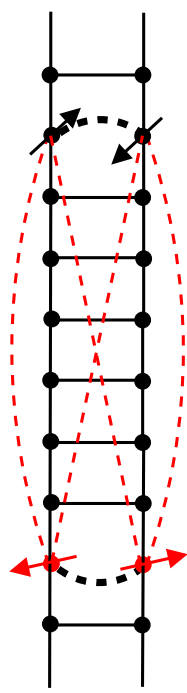

(b)
FIG. 16: (color online) Sketch of a low-energy excitation in the bond-diluted ladder system. (a) Four spins with missing rung bonds are coupled across a clean region of length $l$ with weak effective couplings $J_{\text {eff }} \sim \exp [-\sigma l]$; (b) rotating the spins at one end of the clean region by the same angle, spin excitations occur on the weaker bonds only, leaving the stronger ones unchanged.

What are the excitations leading to the singular response in our model? To answer this question, we focus on the ladder limit, referring to the discussion of bond dilution in ladder systems given in the previous section. There, it was observed that bond dilution leaves free $S=1 / 2$ moments interacting via effective long-range couplings, $J_{\text {eff }}$, exponentially decaying with the distance. The presence of this weak energy scale in the system immediately populates the low-lying spectrum down to the ground state. For any arbitrarily small energy, one can always find two $S=1 / 2$ moments which are sufficiently far from each other to be coupled with this energy. Nonetheless, to be able to excite the system at this energy scale only, one needs the two moments to be separated by a large clean region, such that one can rotate all moments at one end of this region of a given angle, leaving the higher-energy couplings at shorter range unchanged (see Fig. 16). Therefore on the one hand there is an exponentially small energy of the localized excitation, 


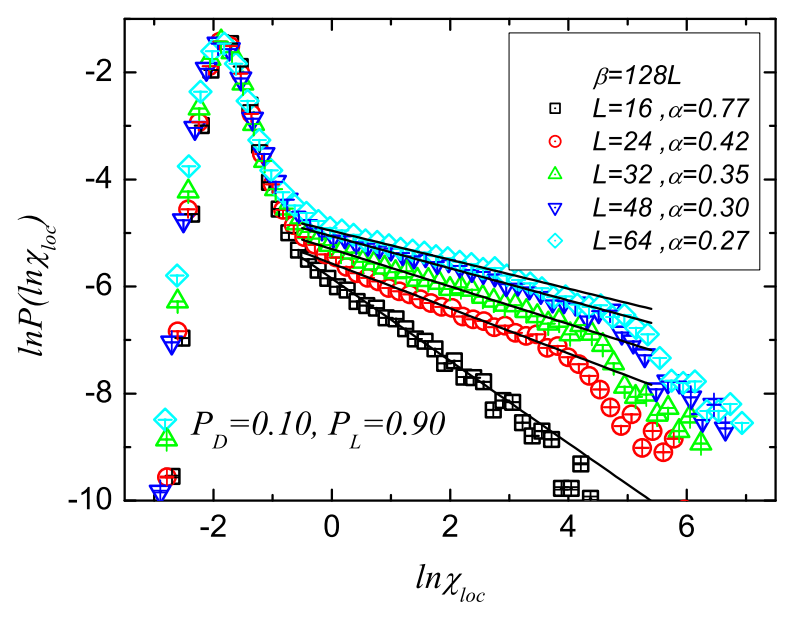

FIG. 17: (color online) Probability distribution of the local susceptibility for a representative point in the quantumdisordered phase $\left(P_{D}=0.15, P_{D}=0.9\right)$, taken at $\beta=128 L$. The slope of the linear tail of the distribution is proportional to the $\alpha$ exponent in the thermodynamic limit (see text).

leading to an equally weak local gap $\Delta$ :

$$
\Delta \sim \exp [-\sigma l]
$$

but, at the same time, the presence of a large clean region of length $l$ between the free moments is a rare event with exponentially small probability

$$
w(l) \sim\left(1-P_{L}\right)^{l} \sim \exp [-c l] .
$$

By substituting Eq. (15) into Eq. (16) with the appropriate metric term to preserve the normalization condition of the probability distribution, the two exponentials compensate each other, leaving a power-law distribution $\underline{46}$ of the local gap $\Delta$ :

$$
w(\Delta) \sim \Delta^{c / \sigma-1} .
$$

A very similar argument can be applied to the dimer limit of the model. Here, e.g., an exponentially rare long clean decorated chain with weakly interacting spins at its ends leads to a similar probability distribution of local gaps.

The above result has immediate consequences for the thermodynamics of the system $\frac{10}{}$, giving rise to the observed power-law behavior of the uniform susceptibility $\chi \sim T^{\alpha-1}$ where $\alpha=c / \sigma$. In particular the constant $c$ is clearly disorder-dependent $\left[c=\left|\ln \left(1-P_{L}\right)\right|\right.$ via Eq. (16)] $]$, such that $\alpha$ is expected to be non-universal, as it is observed in our data.

In order to verify the microscopic interpretation of the QMC results for $\chi_{u, c}$ in terms of a power-law distribution of the local gaps, Eq. (17), we study an observable that directly probes the local gap, i.e. the local susceptibility 4 ,

$$
\chi_{\mathrm{loc}, i} \equiv \int_{0}^{\beta} d \tau\left\langle S_{i}^{z}(\tau) S_{i}^{z}(0)\right\rangle .
$$

Hence, if the $i$-th spin is involved in a local quantum state with a local gap $\Delta$, one obtains in the large- $\tau$ (lowT) limit:

$$
\left\langle S_{i}^{z}(\tau) S_{i}^{z}(0)\right\rangle \sim \exp [-\Delta \tau]
$$

such that, upon imaginary-time integration

$$
\chi_{\mathrm{loc}, i} \sim \frac{1}{\Delta} .
$$

By a simple change of variables in Eq. (17) one then finds a probability distribution for the local susceptibility,

$$
w\left(\chi_{\mathrm{loc}}\right) \sim \chi_{\mathrm{loc}}^{-1-\alpha},
$$

which is more conveniently recast in the form

$$
\ln w\left(\ln \chi_{\mathrm{loc}}\right) \sim-\alpha \ln \chi_{\mathrm{loc}},
$$

i.e. the function $\ln w\left(\ln \chi_{\text {loc }}\right)$ has a tail linear in $\left(\ln \chi_{\text {loc }}\right)$ and a slope that directly yields the exponent $\alpha$.

Fig. 17] shows results for the logarithmic distribution of the local susceptibility, Eq. (22), on sites of the percolating cluster only, for a representative point in the quantum-disordered phase $\left(P_{L}=0.9, P_{D}=0.1\right)$ and for various system sizes at very low temperatures $(\beta=128 L)$. Consistent with the Griffiths-singularity scenario, one observes a linear tail in $\ln w\left(\ln \chi_{\text {loc }}\right)$, reflecting the power-law tail of $w\left(\chi_{\mathrm{loc}}\right)$ for all considered system sizes. This tail shows a strong size dependence, with a decreasing slope for increasing size, and crossing over into a non-linear finite-size tail for large $\chi_{\text {loc }}$ values. The tail of the distribution $w\left(\chi_{\text {loc }}\right)$ is the result of rare events associated with clean regions and exponentially small gaps, such that finite-size corrections significantly affect the tail statistics. Nonetheless, the extracted values of the tail slope $\alpha$ converge towards the range $\alpha \approx 0.2 \div 0.3$, fully consistent with the independent estimate obtained from the low-temperature behavior of the global susceptibility $\chi_{u, c}$ in the ladder limit. This result completes the picture of a quantum Griffiths phase for the quantum-disordered region of the system.

\section{CONCLUSIONS}

In this work, we have investigated the highly nontrivial interplay between quantum fluctuations and geometric disorder realized in the inhomogeneously bonddiluted quantum Heisenberg antiferromagnet on the square lattice. We have observed that the inhomogeneous nature of bond dilution allows for a continuous tuning of 
quantum fluctuations from linear to non-linear. Inhomogeneity enables us to tune the system from a renormalized classical phase, in which the magnetic transition is purely driven by geometric percolation, to a genuine quantum regime in which the magnetic transition is decoupled from the percolation transition. Hence, a novel quantum-disordered phase appears between the magnetic and the percolation transition. This phase has the nature of a quantum Griffiths phase, characterized by finiterange correlations coexisting with a divergent uniform susceptibility.

This represents, to our knowledge, the first evidence of a quantum Griffiths phase in disordered $2 D$ Heisenberg antiferromagnets $\frac{47}{}$, the previous observations being limited so far to one-dimensional Heisenberg antiferromagnets $7,43,44$ and one- and two-dimensional quantum Ising models 10.11 .12 .46 . Given the relevance of the two-dimensional Heisenberg model to the physics of cuprate oxides, we believe that this finding represents an important step towards the possibility of the experimental observation of a quantum Griffiths phase.

Obviously, the most natural form of disorder in real Heisenberg antiferromagnets is site dilution, realized by doping of non-magnetic impurities in the magnetic lattice ${ }^{3}$. Bond dilution can, in principle, be realized by vacancies in the non-magnetic lattice, in particular by vacancies on the sites of the non-magnetic ions involved in the superexchange paths. The presence of such vacancies naturally alters the local electronic structure of the system, such that its effect on the magnetic Hamiltonian may be more elaborate than simple bond dilution, and it might even introduce excess charge carriers in the system. The doping of the non-magnetic ions involved in the superexchange paths with other non-magnetic species introduces instead bond disorder ${ }^{48}$, whose effect can in principle be arbitrarily close to that of bond dilution.

Beside the technical challenges in realizing bond dilution (and bond disorder in general) in real antiferromagnets, a fundamental aspect of this work is the central role of inhomogeneity in the search for novel quantum phases induced by disorder in two-dimensional Heisenberg antiferromagnets. Recent studies 49 show that the Néel ordered state of the square-lattice Heisenberg antiferromagnet is extremely stable towards homogeneous bond disorder, and homogeneous bond dilution destroys Néel order only at the classical percolation threshold ${ }^{4}$. Hence, inhomogeneity is an essential ingredient to realize strong enhancement of quantum effects through disorder beyond the previously investigated scenarios.

\section{ACKNOWLEDGMENTS}

We acknowledge fruitful discussions with L. Balents, N. Bray-Ali, N. Laflorencie, B. Normand, G. Refael, S. Sachdev, H. Saleur and A. Sandvik. S. H. and R. Y. acknowledge hospitality at the KITP, Santa Barbara. This work is supported by the DOE award DE-FG0205ER46240. R. Y. was also supported in part by the NSF through grant No. PHY99-07949. Computational facilities have been generously provided by the HPCCUSC Center.
1 S. Chakravarty, B. I. Halperin, and D. R. Nelson, Phys. Rev. Lett. 60, 1057 (1988).

2 S. Sachdev, Quantum Phase Transitions. Cambridge University Press, Cambridge (1999).

3 O. P. Vajk, P. K. Mang, M. Greven, P. M. Gehring, and J. W. Lynn, Science 295, 1691 (2002).

4 A. W. Sandvik, Phys. Rev. B 66, 024418 (2002).

${ }^{5}$ S. K. Ma, C. Dasgupta, and C. K. Hu, Phys. Rev. Lett. 43, 1434 (1979).

6 D. S. Fisher, Phys. Rev. B 50, 3799 (1994).

7 R. A. Hyman, K. Yang, R. N. Bhatt, and S. M. Girvin, Phys. Rev. Lett. 76,839 (1996); K. Yang, R. A. Hyman, R. N. Bhatt, and S. M. Girvin, J. Appl. Phys. 79, 5096 (1996).

8 E. Manousakis, Rev. Mod. Phys. 63, 1 (1991).

9 A direct transition from an ordered phase into an unconventional quantum disordered phase driven by randomness has been demonstrated so far only in the case of random quantum Ising models in a transverse field, either in one or two dimensions. See, e.g., Refs. 10, 11, 12.

10 D. S. Fisher, Phys. Rev. Lett. 69, 534 (1992); Phys. Rev. B 51, 6411 (1995).

11 T. Senthil and S. Sachdev, Phys. Rev. Lett. 77, 5292 (1996).
12 C. Pich, A. P. Young, H. Rieger, and N. Kawashima, Phys. Rev. Lett 81, 5916 (1998).

13 A. L. Chernyshev, Y. C. Chen, and A. H. Castro Neto, Phys. Rev. B 65, 104407 (2002).

14 E. R. Mucciolo, A. H. Castro Neto, and C. Chamon, Phys. Rev. B 69214424 (2004).

15 N. Bray-Ali and J. E. Moore, Phys. Rev. B 69, 184505 (2004).

16 M. Corti, A. Rigamonti, F. Tabak, P. Carretta, F. Licci, and L. Raffo, Phys. Rev. B 52, 4226 (1995).

17 P. Carretta, A. Rigamonti, and R. Sala Phys. Rev. B 55, 3734 (1997).

18 D. Stauffer and A. Aharony Introduction to Percolation Theory. Taylor and Francis, London (1994).

19 K. Kato, S. Todo, K. Harada, N. Kawashima, S. Miyashita, and H. Takayama, Phys. Rev. Lett. 84, 4204 (2000).

20 S. Todo, C. Yasuda, K. Kato, K. Harada, N. Kawashima, S. Miyashita, and H. Takayama, Prog. Theor. Phys. Suppl. 138, 507 (2000).

21 C. Yasuda, S. Todo, K. Harada, N. Kawashima, S. Miyashita, and H. Takayama, Phys. Rev. B 63, R140415 (2001).

22 A recent study (T. Vojta and J. Schmalian, Phys. Rev. Lett. 95, 237206 (2005)) shows that only the critical ex- 
ponents $\beta$ and $\nu$ coincide with those $\left(\beta_{\mathrm{cl}}=5 / 36\right.$ and $\left.\nu_{\mathrm{cl}}=4 / 3\right)$ of the classical universality class, while the other exponents are shifted $\left(\alpha=\alpha_{\mathrm{cl}}-\nu z, \gamma=\gamma_{\mathrm{cl}}+\nu z\right.$, $\delta=\delta_{\mathrm{cl}}+\nu z / \beta$, and $\left.\eta=\eta_{\mathrm{cl}}-z\right)$ due to the appearence of the dynamical critical exponent in the scaling relations (see, e.g., M. Continentino, Quantum scaling in Many-Body Systems, World Scientific, Singapore, 2001). Nonetheless it is interesting to notice that the experimentally accessible exponents, through e.g. neutron scattering, are the unshifted ones, $\beta$ and $\nu$, along with the critical exponent of the static structure factor $\gamma_{S}=\gamma-\nu z$ which concides with the percolation exponent $\gamma_{\mathrm{cl}}$.

23 R. Yu, T. Roscilde, and S. Haas, Phys. Rev. Lett. 94, 197204 (2005).

24 A. W. Sandvik, Phys. Rev. Lett. 89, 177201 (2002).

25 O. P. Vajk and M. Greven, Phys. Rev. Lett. 89, 177202 (2002).

26 R. Sknepnek, T. Vojta, and M. Vojta, Phys. Rev. Lett. 93, 097201 (2004).

27 A short, partial account of this work has appeared previously ${ }^{23}$.

28 R. B. Griffiths, Phys. Rev. Lett. 23, 17 (1969).

29 B. M. McCoy, Phys. Rev. Lett. 23, 383 (1969); Phys. Rev. 188, 1014 (1969).

30 E. Dagotto and T. M. Rice, Science 271, 619 (1996).

31 J. Igarashi, T. Tonegawa, M. Kaburagi, P. Fulde, Phys. Rev. B 51, 5814 (1995).

32 S. Moukouri, Liang Chen, and L. G. Caron, Phys. Rev. B 53, R488 (1996).

33 G. Sierra, M. A. Martin-Delgado, S.R. White, D.J. Scalapino, J. Dukelsky, Phys. Rev. B 59, 7973 (1999).

34 S. R. White, R. M. Noack, and D. J. Scalapino, Phys. Rev. Lett. 73, 886 (1994).

35 O. F. Syljuåsen and A. W. Sandvik, Phys. Rev. E 66, 046701 (2002).

36 M. E. J. Newman and R. M. Ziff, Phys. Rev. Lett. 85, 4104 (2000); M. E. J. Newman and R. M. Ziff, Phys. Rev. E 64, 016706 (2001).

37 F. Cooper, B. Freedman, and D. Preston, Nucl. Phys. B
210, 210 (1982)

38 H. Fukuyama, N. Nagaosa, M. Saito, and T. Tanimoto, J. Phys. Soc. Jpn. 65, 2377 (1996).

39 H.-J. Mikeska, U. Neugebauer, and U. Schollwöck, Phys. Rev. B 55, 2955 (1997).

40 M. Sigrist and A. Furusaki, J. Phys. Soc. Jpn. 65, 2385 (1996).

41 M. Greven and R. J. Birgeneau, Phys. Rev. Lett. 81, 1945 (1998).

42 C. Yasuda, S. Todo, M. Matsumoto, and H. Takayama, Phys. Rev. B 64, 092405 (2001).

43 R. A. Hyman and K. Yang, Phys. Rev. Lett. 78, 1783 (1997).

44 S. Todo, K. Kato, and H. Takayama, J. Phys. Soc. Jpn. Suppl. 69 A, 355 (2000).

45 F. Iglói and C. Monthus, Phys. Rep. 412, 277 (2005).

46 H. Rieger and A. P. Young, Phys. Rev. B 54, 3328 (1996).

47 A real-space renormalization group study of the dimerized 2D Heisenberg antiferromagnet with bond disorder (Y.-C. Lin, R. Mélin, H. Rieger, and F. Iglói, Phys. Rev. B 68, 024424 (2003)) suggests the occurrence of a quantum Griffiths phase in the dimerized 2D Heisenberg antiferromagnet with bond disorder. Nonetheless, a detailed quantum Monte Carlo study of the above model in presence of bond dilution (C. Yasuda, S. Todo, M. Matsumoto, and H. Takayama, J. Phys. Chem. Solids 63, 1607 (2002); C. Yasuda, S. Todo, M. Matsumoto, and H. Takayama, Prog. Theor. Phys. Suppl. 145, 339 (2002)) shows that the gapped dimer-singlet phase of the clean system is stable to disorder, and it is driven to a gapless antiferromagnetic phase at a finite value of bond dilution through an order-by-disorder mechanism.

48 A. Oosawa and H. Tanaka, Phys. Rev. B 65, 184437 (2002); T. Masuda, A. Zheludev, K. Uchinokura, J.-H. Chung, and S. Park, Phys. Rev. Lett. 93, 077206 (2004).

49 N. Laflorencie, S. Wessel, A. Laeuchli, H. Rieger, cond-mat/0511306 